\title{
Potential Benefits of a Selective Region High-frequency Diathermy with Therapeutic Exercises on Older Persons with Degenerative Knee Osteoarthritis: A Case Report
}

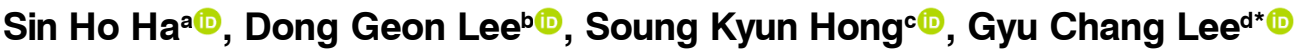

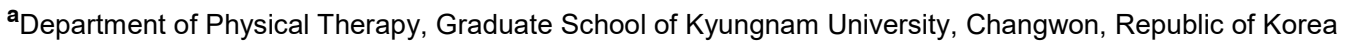 \\ ${ }^{b}$ Department of Physical Therapy, Sinsegye Nursing Care Facility, Changwon, Republic of Korea \\ 'Department of Physical Therapy, Woosuk University, Wanju, Republic of Korea \\ ${ }^{\mathrm{d}}$ Department of Physical Therapy, Kyungnam University, Changwon, Republic of Korea
}

Objective: The purpose of this case study was to investigate selective region high-frequency diathermy at trigger points with therapeutic exercises on pain, function, balance and gait in older patients with degenerative knee osteoarthritis (DKO).

Design: A case report.

Methods: The patient who participated in this study was a 71-year-old woman, who had been diagnosed with moderate osteoarthritis with grade II Kellgren \& Lawrence grading scale. The intervention consisted of selective region high-frequency diathermy at trigger points, with hip and knee stretching and strengthening exercises. The participant was given assessments before and after every intervention session using the Visual Analogue Scale (VAS), Western Ontario and McMaster Universities Arthritis Index (WOMAC), the Timed Up and Go test (TUG) and the 10 Meter Walk Test (10MWT). The participant performed the intervention 18 times for a total of 30 minutes each.

Results: As a result of this study, the patient VAS decreased to 3 points, and the WOMAC decreased to 53 points. In addition, the TUG decreased to $3.25 \mathrm{~s}$ and the 10MWT decreased to $1.14 \mathrm{~s}$.

Conclusions: The results of this study suggest that selective region high-frequency diathermy at trigger points with therapeutic exercises may be an effective intervention to decrease pain, improve knee function, balance and gait in patients with DKO. The selective region high-frequency diathermy with therapeutic exercises may be feasible and provide potential benefits for rehabilitation of DKO.

Key Words: Knee osteoarthritis, Diathermy, Elderly

\section{Introduction}

Musculoskeletal degenerative diseases, such as osteoporosis, osteoarthritis and spondylolysis often occur in the elderly [1]. Among them, osteoarthritis occurs the most often. In fact, the prevalence of degenerative diseases occurring in the knees of people over 40 years old is as high as 15-45\% [2,3]. Degenerative osteoarthritis causes joint deformity with irregular articular surfaces, and therefore, pain and joint stiffness occurs [4]. Both the quadriceps and hamstring muscles are shortened due to the thickening of the knee articular capsule, which results in the reduction in the range of motion and an increase in viscoelasticity and contractibility of the soft tissues [5-8]. In addition, joint function is undermined as activities are reduced due to joint deformity caused by continuous impact. Following this, lower extremity performing motions including gait are limited [9], hence physical activities are decreased, causing an

Received: Jul 15, 2021 Revised: Oct 7, 2021 Accepted: Oct 15, 2021

Corresponding author: Gyu Chang Lee (ORCID https://orcid.org/0000-0001-6404-203X)

Department of Physical Therapy, Kyungnam University, Changwon, Republic of Korea

Tel: +82-55-249-2739 Fax: +82-505-999-2173 E-mail: leegc76@hanmail.net

This is an Open-Access article distributed under the terms of the Creative Commons Attribution Non-Commercial License (http://creativecommons.org/licenses/ by-nc/4.0) which permits unrestricted non-commercial use, distribution, and reproduction in any medium, provided the original work is properly cited.

Copyright () 2021 Korean Academy of Physical Therapy Rehabilitation Science 
inconvenience on a daily basis [4].

The intervention for degenerative knee osteoarthritis includes surgical options such as joint replacement, and non-surgical options such as medicinal and physical therapy, which alleviate inflammation using injections or medications. These methods are applied by considering the patient's condition and any risk [9]. Physical therapy methods include manual therapy, therapeutic exercise, taping, electrotherapy, and light therapy, among others [10].

High-frequency diathermy has been used as a physical therapy method. It generates a safe range of frictional heat deep inside the body without any burn risks, and does not stimulate sensory and motor nerves due to its very short pulse duration. The short pulse duration also does not induce muscle contraction during the diathermic process while increasing the temperature of local tissues, and thereby, reducing pain [11]. Therapeutic exercise plays an important role in managing osteoarthritis, and is more advantageous if doing exercise with weight loss, and direct guidance by a therapist can help reduce pain rather than a home program, and muscle strengthening helps prevent knee osteoarthritis [12]. In particular, the simultaneous application of both high-frequency diathermy and therapeutic exercise further alleviates the pain of patients with degenerative knee osteoarthritis and is effective in improving the function levels [13], joint stiffness, balance [14] and gait [15]. In addition, applying diathermy and therapeutic exercise together to patients with knee pain with swelling further reduces pain, and improves physical function and quality of life [16].

However, the effect of intervention on both diathermy and therapeutic exercise has not yet been clearly concluded. In a study [17], they suggested that the intervention using both high-frequency diathermy and therapeutic exercise cannot result in better improvement of degenerative knee osteoarthritis, than intervention with only therapeutic exercise. There is also a systematic review which cannot show that the intervention using both high-frequency diathermy and therapeutic exercise is more effective for functional performance, than using therapeutic exercise alone [18].

Trigger points, irregular and sensitive tubercles discovered in strained muscles, induce paresthesia, referred pain and impaired motor function [19], often occurring in tendons of muscles and can be caused by muscle tension, myositis, arthritis, motor abnormality and direct trauma [20]. The trigger points of quadriceps muscles result in pain at the front of the knees and around the kneecaps [21] and can involve pain and dysfunction experienced by patients with knee osteoarthritis [22]. The intervention applied to such trigger points is effective in recovering the function of quadriceps muscles [21]. Pain and sensitivity of trigger points can also be reduced by applying high-frequency diathermy to these points [23].

The electrodes of short-wave diathermy which are often used, are usually applied to relatively wide body parts due to their size, so selective diathermy for trigger points on muscles may be impossible. If electrodes with which high-frequency diathermy can be selectively applied to trigger points with the application of therapeutic exercise at the same time, better results may be expected. It was reported that pain, disability index, and movement was improved when suboccipital release technique techniques and selective regulation high-frequency diathermy were applied to patients with kinetic tension headache [24], as a result of applying high-frequency diathermy to the pain area of chronic low back pain patients, the pain and disability index decreased [25]. It was also reported that it is effective in increasing range of motion and reducing muscle tension and muscle stiffness when applied to stiffen muscles [26]. Although trigger points are involved with the pain and dysfunction of patients with degenerative knee osteoarthritis and can also reduce pain by increasing the pain threshold, there have been no studies on the simultaneous application of high-frequency diathermy and therapeutic exercise to these trigger points of patients and the effects thereof.

Therefore, in this study, we investigated the feasibility and potential benefits of selective region high-frequency diathermy at trigger points with therapeutic exercises on the pain, dysfunction, balance, and gait of a patient with degenerative knee osteoarthritis.

\section{Case Report}

\section{History and systems review}

The patient who participated in this study was a 
71-year-old female with a height of $157.32 \mathrm{~cm}$ and a weight of $68.15 \mathrm{~kg}$, which had been consistently maintained for 10 years. Her left limbs were dominant.

She visited a local hospital eight years back because she started experiencing pain in her knees and was subsequently diagnosed with knee osteoarthritis; since then, she has received physical therapy intermittently. For the last three years, her knee pain has worsened, especially in her left knee, wherein it has significantly increased. The participant visited the $\mathrm{C}$ hospital outpatient clinic and she was diagnosed with moderate osteoarthritis of the left knee (grade II Kellgren \& Lawrence grading scale), and she was referred to physical therapy.

The participant had no musculoskeletal, nervous and mental diseases, except for degenerative knee osteoarthritis and had not undergone any surgical or medicinal interventions for this condition.

Her rehabilitative goal was to reduce pain, walk comfortably up the stairs and do housework easily.

\section{Tests \& Measures}

The participant was examined the pain, functional status, balance, and gait of the subject a total of 19 times, once before and after each intervention session for 6 weeks. Each examination completed was using the Visual Analogue Scale (VAS), Western Ontario and McMaster Universities Arthritis Index (WOMAC), Timed Up and Go test (TUG) and 10 Meter Walk Test (10MWT), respectively.

The participant's pain was examined by using VAS, recorded the pain in her left knee and asked her to check the pain by answering a questionnaire. VAS is considered a reliable $(\mathrm{ICC}=0.99)$ and valid pain scale [27].

WOMAC was used to examine the knee function. WOMAC is a questionnaire for evaluating the pain, stiffness and dysfunction of patients with arthritis, and consists of a total of 24 questions including 5 items for pain; 2 items for joint stiffness; and 17 items for difficulty of daily life performance. WOMAC is based on a 5 points scale (0-4 points) with a total score of 96 : none $=0 ;$ a little $=1$; average $=2$; serious $=3$; and very serious $=4$. Higher total scores are associated with the worsening of symptoms and more limited activities. This tool's reliability and validity were affirmed [28]
TUG was used to examine balance and the participant was asked to start sitting down in a chair with armrests, get up at the sound of a starting signal, walk towards a point $3 \mathrm{~m}$ away, then return and again sit down in the chair. TUG has an advantage in that it can rapidly measure mobility and dynamic balance, with a test-retest reliability of $\mathrm{ICC}=0.96$ [29].

Lastly, the 10MWT was used to examine her gait ability and asked her to walk along a line with the length of $10 \mathrm{~m}$, which was marked on the floor, at a convenient speed. This test has a retest reliability of $\mathrm{ICC}=0.93[30]$.

\section{Clinical impression}

The participant was diagnosed with moderate degenerative knee osteoarthritis of the left knee (grade II on Kellgren \& Lawrence grading scale), and reported pain in the left knee. Although the participant did not use an assistive tool for gait, she reported serious difficulty in walking up stairs and her performance was limited due to pain in one leg when standing. Her pre-assessment scores were as follows: $\mathrm{VAS}=5$ (indicating serious pain), $\quad$ WOMAC $=66$ (indicating the patient's knee function had seriously deteriorated due to the degenerative knee osteoarthritis), $\quad \mathrm{TUG}=12.38$ seconds (indicating the moderate reduction of balance), and $10 \mathrm{MWT}=6.29$ seconds (indicating pain in her left knee during walking).

\section{Intervention}

Winback 3SE (Back 3SE, Winback, France), a high-frequency diathermy developed by WINBACK was used for the selective region control high-frequency diathermy (Figure 1). Winback 3SE can adjust the depth of infiltration, as it is able to select a variety of frequencies such as $300 \mathrm{KHz}, 500 \mathrm{KHz}, 1 \mathrm{MHz}$, etc. With it, the precise diathermy of injured regions is possible, since physical therapists can directly handle regions to which heat is transferred with their hands. This is an advantage as both high-frequency diathermy and therapeutic exercise can be applied to patients simultaneously. In this study, it utilized the frequency of $500 \mathrm{KHz}$ during every intervention session and used 


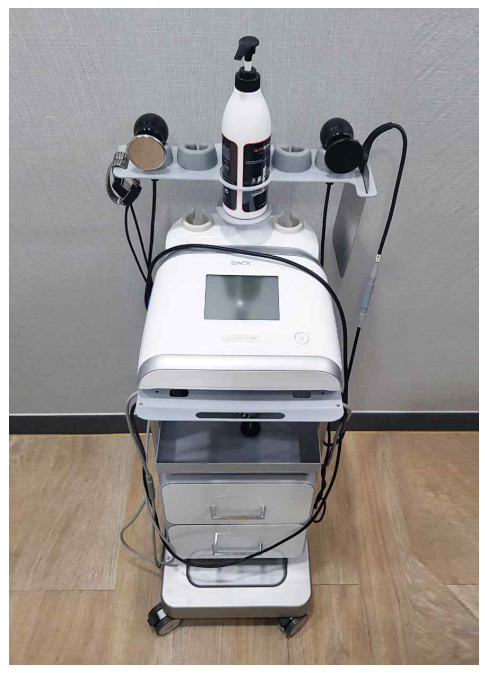

Figure 1. High-frequency diathermy

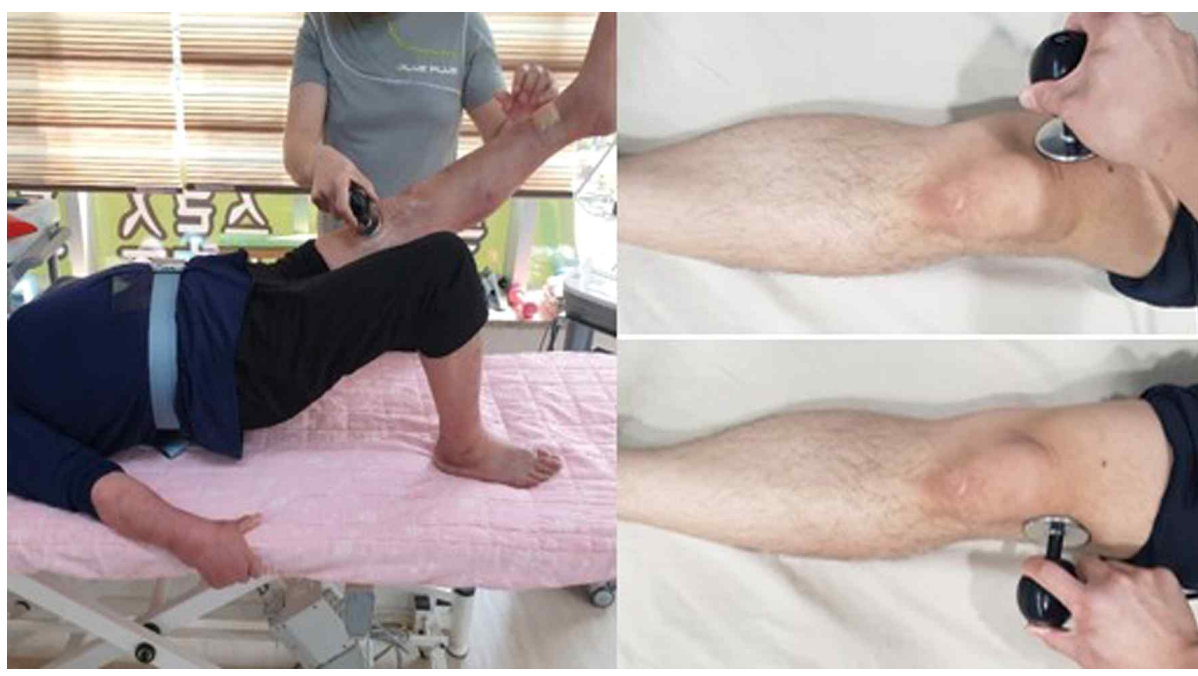

Figure 2. Selective region high-frequency diathermy and therapeutic exercise

and electrode (Winback 3SE)

resistive electric transfer (RET) to facilitate the diathermy of muscles. The intensity was gradually increased from $10 \%$ to the heat level that the patient could endure $(0 \%-100 \%)$, with an average of $50 \%$ [25]. The regions to which the intervention was applied included the quadriceps and hamstring muscles. For applying quadriceps muscles, the diathermy was conducted by attaching fixed electrodes to and applying trigger points to regions below the waist, while for hamstring muscles, it was implemented by attaching fixed electrodes to and applying trigger points to those below the abdomen. The selective region control high-frequency diathermy was also applied to these muscles during the therapeutic exercise (Figure 2).

According to the study by Kuru et al. [31], they examined the effects of therapeutic exercise applied to patients with knee osteoarthritis, including bridge exercise and squat, which strengthen the knee muscles and enhance their flexibility. It gradually increased the exercise intensity by changing the number of the exercises as well as the number of repetitions, and the manual resistance, depending on the participant's condition and performance (Table 1).

The selective region control high-frequency diathermy was applied along with the therapeutic exercise, and the participant received a total of 18 sessions of the intervention: one 30 minute session, three sessions a week, for 6 weeks.

\section{Results}

The results of pain, knee function, balance, and gait are shown in Table 2. The scores on VAS were 5 in the pre-assessment before the intervention. After 9 sessions of the intervention the score remained 5; and only decreased to 2 after the last intervention. This indicates that there was no difference in the score between the pre-assessment and the assessment after 9 sessions, and the score was decreased by $3(60 \%)$ only in the assessment after the last session (Table 3).

The scores on WOMAC Index were 66 in the pre-assessment; 41 after 9 sessions; and 13 after the last intervention session, indicating that the score decreased by $25(37.88 \%)$ after 9 sessions, and by 53 $(80.3 \%)$ after the last session (Table 2). 
Table 1. Therapeutic exercises

\begin{tabular}{|c|c|c|}
\hline Week & Therapeutic exercises & Intensity \\
\hline \multirow{6}{*}{1 week } & Quadriceps muscle isometric contraction in sitting & 5 seconds hold, 8 repetitions \\
\hline & Terminal knee extension $(0-30)$ in sitting & 10 repetitions, 3 sets \\
\hline & Isometric hip abduction in sitting & 5 seconds hold, 8 repetitions \\
\hline & Bridge exercise in supine position & 5 seconds hold, 8 repetitions \\
\hline & Quadriceps muscle stretching in prone position & 10 seconds hold, 5 repetitions \\
\hline & Hamstring muscle stretching in sitting & 10 seconds hold, 5 repetitions \\
\hline \multirow{6}{*}{2 week } & Quadriceps muscle isometric contraction in sitting & 5 seconds hold, 8 repetitions \\
\hline & Terminal knee extension $(0-30)$ in sitting & 10 repetitions, 3 sets \\
\hline & Isometric hip abduction in sitting & 10 seconds hold, 10 repetitions \\
\hline & Bridge exercise in supine position & 10 seconds hold, 10 repetitions \\
\hline & Quadriceps muscle stretching in prone position & 10 seconds hold, 5 repetitions \\
\hline & Hamstring muscle stretching in sitting & 10 seconds hold, 5 repetitions \\
\hline \multirow{6}{*}{3 week } & Quadriceps muscle isometric contraction in sitting & 10 seconds hold, 10 repetitions \\
\hline & Terminal knee extension $(0-20)$ in sitting & 10 repetitions, 3 sets \\
\hline & Straight leg extension in sitting & 10 repetitions, 3 sets \\
\hline & Straight leg extension with bridge exercise & 10 repetitions, 3 sets \\
\hline & Quadriceps muscle stretching in prone position & 10 seconds hold, 5 repetitions \\
\hline & Hamstring muscle stretching in sitting & 10 seconds hold, 5 repetitions \\
\hline \multirow{7}{*}{4 week } & Quadriceps muscle isometric contraction in sitting & 10 seconds hold, 5 repetitions \\
\hline & Terminal knee extension $\left(0-10^{\circ}\right)$ in sitting & 10 repetitions, 3 sets \\
\hline & Straight leg extension in sitting & 10 repetitions, 3 sets \\
\hline & Straight leg extension with bridge exercise & 10 repetitions, 3 sets \\
\hline & Squats to $30^{\circ}$ knee flexion combined with gluteal muscle contractions & 10 seconds hold, 5 repetitions \\
\hline & Quadriceps muscle stretching in prone position & 10 seconds hold, 5 repetitions \\
\hline & Hamstring muscle stretching in sitting & 10 seconds hold, 5 repetitions \\
\hline \multirow{7}{*}{5 week } & Terminal knee extension $\left(0-10^{\circ}\right)$ in sitting & 10 repetitions, 3 sets \\
\hline & Straight leg extension in sitting & 10 repetitions, 3 sets \\
\hline & Straight leg extension with bridge exercise & 10 repetitions, 3 sets \\
\hline & Squats to $60^{\circ}$ knee flexion combined with gluteal muscle contractions & 10 repetitions, 3 sets \\
\hline & Quadriceps muscle stretching in prone position & 10 seconds hold, 5 repetitions \\
\hline & Hamstring muscle stretching in sitting & 10 seconds hold, 5 repetitions \\
\hline & Iliotibial tract stretching while standing & 10 seconds hold, 5 repetitions \\
\hline \multirow{8}{*}{6 week } & Straight leg extension in sitting Straight leg extension with & 10 repetitions, 3 sets \\
\hline & bridge exercise & 10 repetitions, 3 sets \\
\hline & Squats to $60^{\circ}$ knee flexion combined with gluteal muscle contractions & 10 repetitions, 3 sets \\
\hline & Walking with dorsiflexed ankle & 10 seconds hold, 5 repetitions \\
\hline & Quadriceps muscle stretching in prone position & 10 seconds hold, 5 repetitions \\
\hline & Hamstring muscle stretching in sitting & 10 seconds hold, 5 repetitions \\
\hline & Iliotibial tract stretching while standing & 10 seconds hold, 5 repetitions \\
\hline & Gastrocnemius muscle stretching against to wall while standing & 10 seconds hold, 5 repetitions \\
\hline
\end{tabular}


Table 3. Outcome on the pain, functional status, balance, and gait

\begin{tabular}{llll}
\hline Variables/sessions & Pre & 9 sessions (3 week) & 18 sessions (6 week) \\
\hline VAS (point) & 5 & 5 & 2 \\
\hline K-WOMAC (point) & 66 & 41 & 13 \\
\hline TUG (sec) & 12.38 & 10.84 & 9.13 \\
\hline 10MWT (sec) & 6.29 & 5.79 & 5.15 \\
\hline
\end{tabular}

VAS: Visual Analogue Scale, K-WOMAC: Korean version of Western Ontario and McMaster Universities Arthritis Index, TUG: Timed Up and Go test, 10MWT: 10 Meter Walk Test.

The TUG test was 12.38 seconds in the pre-assessment; 10.84 seconds after 9 sessions; and 9.13 seconds after the last intervention session, indicating that it decreased by 1.54 seconds $(12.44 \%)$ after 9 sessions, and by 3.25 seconds (26.25\%) after the last session (Table 2).

The 10MWT was 6.29 seconds in the pre-assessment; 5.79 seconds after 9 sessions; and 5.15 seconds after the last session, indicating that it decreased by 0.5 seconds $(7.95 \%)$ after 9 sessions and by 1.14 seconds $(18.12 \%$ ) after the last session (Table 2).

\section{Discussion}

This study investigated the effect of selective region control high-frequency diathermy at trigger points with therapeutic exercise on the pain, dysfunction, balance, and gait of patients with degenerative knee osteoarthritis through a case report. The findings showed that a total of 18 sessions of the intervention resulted in the reduction of pain and the enhancement of dysfunction, balance and gait, compared to before the intervention sessions.

Cetin et al. [13] applied both high-frequency diathermy and therapeutic exercise to patients with degenerative knee osteoarthritis at three sessions a week, for 8 weeks, and the findings showed that the pain of one group of participants to which both of therapies were applied was more reduced than that of the other group to which only the therapeutic exercise was applied. This study also demonstrated that the scores on VAS were reduced by $60 \%$ after the last intervention session, compared to before the first. The result of this case study is consistent with that of Cetin et al. [13] showing that the application of both high-frequency diathermy and therapeutic exercise resulted in better improvement of the pain of patients with degenerative knee osteoarthritis, than the application of only the therapeutic exercise. Heat increases the blood flow [32], and therefore, alleviates the pain in patients with osteoarthritis [33] by dilating blood vessels and increasing the permeability of capillaries, cell metabolic rates and the expansibility of collagen, reduces muscle shrinkage and boosts nerve conduction. It might reduce the pain, since exercises for strengthening the muscles around knees has been known to decrease pain [34]. In addition, although the trigger points of quadriceps muscles worsen the pain of patients with degenerative knee osteoarthritis and result in dysfunction [22], the application of high-frequency diathermy reduced the pain [23]. From this we have seen the pain seemed to have greater reductions by selectively applying the high-frequency diathermy to only the trigger points. In a study by Lee et al., it was reported that the pain and movement was improved more than only the suboccipital release technique applied as a result of simultaneous application of suboccipital release technique and high-frequency diathermy. [24]. In the present study, since selective region high-frequency diathermy at trigger points with therapeutic exercises were performed, the pain of the degenerative knee osteoarthritis was reduced. In a study by Kwon et al., high-frequency diathermy was applied to areas where pain was felt in the chronic low back pain, and as a result, pain and oswestry disability index decreased. [25]. In the present study, selective region high-frequency diathermy was applied to quadriceps and hamstring where the knee osteoarthritic patient felt pain, resulting in reduced pain and improved WOMAC index. In this study, the scores on the WOMAC index was reduced by 
$80.3 \%$ after the last intervention session, compared to before the first, indicating the improvement of knee function. The result is likely to be similar to Rabini et al. [35] demonstrating that the high-frequency diathermy decreases the scores of patients with degenerative knee osteoarthritis. The joint pain results in hypoesthesia and reduces various muscles' functional motion [36]; knee pain, for example, weakens the strength of quadriceps muscles [37]. Patients with degenerative knee osteoarthritis can alleviate pain, enhance the benefits of weight training and improve their functional status, through the combination of high-frequency diathermy and therapeutic exercises. These benefits are derived through the therapeutic exercise improving the joint symptoms and enhance their motion [38], while the heat increases their knees' range of motion [39] and enhances the benefits of weight training [40]. Moreover, the pain caused by trigger points result in the dysfunction of lower limbs of these patients [41], thus the function of lower limbs would to be improved by treating trigger points, and thereby resolving referred pain.

Giombini et al. [14] divided 60 patients with degenerative knee osteoarthritis into experiment and control groups, and then applied the high-frequency diathermy to the former and attempted to verify the placebo effect from the latter. The findings showed that the TUG was significantly improved in the experimental group. In this study, the TUG was also decreased by $26.25 \%$ overall, corresponding to Giombini et al. [14], demonstrating that the balance of patients with degenerative knee osteoarthritis is improved by high-frequency diathermy. There is usually a prominent reduction of the dynamic balance of patients who suffer from this condition [42], this reduction of balance is closely related with pain felt in the knees [43]. This effect is especially more prominently reduced, as pain of the quadriceps muscle increases [44], hence, the improvement of this pain is important for enhancing balance. In addition, the increase of both quadriceps and hamstring muscle strength significantly enhances the dynamic balance and decreases the risk of falling [45], while the decrease of the hamstring muscle's flexibility induces the imbalance of main muscles [46]. Physical balance can be, therefore, maintained only after the flexibility of the hamstring muscles is enhanced. In a study by Kim et al., when high-frequency diathermy was applied to 24 subjects with stiffness in hamstring for 15 minutes, the active knee extension range of motion increased and muscle tension and muscle stiffness decreased. [26]. Therefore, in the present study, high-frequency diathermy was applied to trigger points to reduce knee pain, muscle stiffness, and muscle stiffness, increasing range of motion, and improving balance because of the increased muscle strength and flexibility by therapeutic exercises. According to Ozen et al. [15], the high-frequency diathermy decreases pain, improving the functional status and gait. In the present study, the 10MWT was decreased by $18.12 \%$ overall which corresponds to Ozen et al. [15], demonstrating that the high-frequency diathermy enhances gait. The pain results in the disruption of normal gait patterns [47], function is especially limited with the pain occurring in one leg when standing [48]. Reduction of gait speed is a compensatory strategy for decreasing the load on the knee joints [49], which is imposed on knees [50] and muscle activities through the normal motion of the knees [51]. Patients with degenerative knee osteoarthritis, therefore, try to limit or even avoid walking altogether, as a strategy for managing their symptoms [52]. Hence, the reduction of pain may play an important role in enhancing the walking ability of patients this would also be improved by enhancing standing which otherwise may be limited due to the pain in one or both legs.

In addition, the weakening of the quadriceps muscles is one of the symptoms that prominently appears in patients with degenerative knee osteoarthritis [53]. The strength of the quadriceps muscles is associated with pain and gait [54], so the strengthening of quadriceps muscles is important for maintaining gait. In the present study, it reduced the pain by applying the high-frequency diathermy to the trigger points of patients with degenerative knee osteoarthritis, this results in the enhancement of weight bearing, while the increase of muscle activities enhances the gait. Moreover, gait is also thought to be enhanced, through the strengthening of quadriceps muscles with the help of the therapeutic exercise which facilitates weight bearing during walking.

The findings showed that the selective region 
control high-frequency diathermy at trigger point with therapeutic exercise may have a positive effect on the improvement in the pain, knee function, balance and gait of patients with degenerative knee osteoarthritis. This is a case study on only one patient with degenerative knee osteoarthritis; therefore, the findings cannot be generalized. Further studies should thus continue to verify the effects of this intervention combination a high quality study with larger sample sizes..

The findings of this case study showed the reduction of pain and the enhancement of knee function, balance and gait. The selective region high-frequency diathermy at trigger point with therapeutic exercise would be an effective intervention for patients with degenerative knee osteoarthritis, who have pain in their knees or whose knee function, balance, and gait have deteriorated.

\section{Conclusion}

The findings of this case study showed the reduction of pain and the enhancement of knee function, balance and gait after applying the selective region high-frequency diathermy at trigger point with therapeutic exercise. Thus, the intervention would be an effective for patients with degenerative knee osteoarthritis. The selective region high-frequency diathermy with therapeutic exercises may be feasible and provide potential benefits for rehabilitation of degenerative knee osteoarthritis.

\section{Acknowledgements}

Not applicable.

\section{Conflict of interest}

The authors declare that they have no competing interests.

\section{References}

1. Imagama $\mathrm{S}$, Ando $\mathrm{K}$, Kobayashi $\mathrm{K}$, Seki $\mathrm{T}$, Hamada $\mathrm{T}$, Machino $\mathrm{M}$, et al. Impact of comorbidity rates of lumbar spondylosis, knee osteoarthritis, and osteoporosis on physical QOL and risk factors for poor physical QOL in middle-aged and elderly people. Mod Rheumatol. 2020;30:402-9.

2. Brooks PM. Impact of osteoarthritis on individuals and society: how much disability? Social consequences and health economic implications. Curr Opin Rheumatol. 2002;14:573-7.

3. Scott D, Kowalczyk A. Osteoarthritis of the knee. BMJ Clin Evid. 2007;2007:1121.

4. Davies CM, Guilak F, Weinberg JB, Fermor B. Reactive nitrogen and oxygen species in interleukin-1-mediated DNA damage associated with osteoarthritis. Osteoarthritis Cartilage. 2008;16:624-30.

5. Ersoz M, Ergun S. Relationship between knee range of motion and Kellgren-Lawrence radiographic scores in knee osteoarthritis. Am J Phys Med Rehabil. 2003;82:110-5.

6. Holla JF, Steultjens MP, van der Leeden M, Roorda LD, Bierma-Zeinstra SM, den Broeder AA, et al. Determinants of range of joint motion in patients with early symptomatic osteoarthritis of the hip and/or knee: an exploratory study in the CHECK cohort. Osteoarthritis Cartilage. 2011;19:411-9.

7. Ozdemir F, Tukenmez O, Kokino S, Turan FN. How do marginal osteophytes, joint space narrowing and range of motion affect each other in patients with knee osteoarthritis. Rheumatol Int 2006;26:516-22.

8. Simons DG, Mense S. Understanding and measurement of muscle tone as related to clinical muscle pain. Pain. 1998;75:1-17.

9. Zhang W, Nuki G, Moskowitz RW, Abramson S, Altman RD, Arden NK, et al. OARSI recommendations for the management of hip and knee osteoarthritis: part III: Changes in evidence following systematic cumulative update of research published through January 2009. Osteoarthritis Cartilage. 2010;18:476-99.

10. Vicenzino B, Paungmali A, Buratowski S, Wright A. Specific manipulative therapy treatment for chronic lateral epicondylalgia produces uniquely characteristic hypoalgesia. Man Ther. 2001;6:205-12.

11. Ganzit GP. New methods in the treatment of jointmuscular pathologies in athletes: The "TECAR" therapy. Medicina Dello Sport. 2000;53:361-7.

12. Bennell K, Hinman R. Exercise as a treatment for osteoarthritis. Curr Opin Rheumatol. 2005;17:63440 . 
13. Cetin N, Aytar A, Atalay A, Akman MN. Comparing hot pack, short-wave diathermy, ultrasound, and TENS on isokinetic strength, pain, and functional status of women with osteoarthritic knees: a single-blind, randomized, controlled trial. Am J Phys Med Rehabil. 2008;87:443-51.

14. Giombini A, Di Cesare A, Di Cesare M, Ripani M, Maffulli N. Localized hyperthermia induced by microwave diathermy in osteoarthritis of the knee: a randomized placebo-controlled double-blind clinical trial. Knee Surg Sports Traumatol Arthrosc. 2011;19:980-7.

15. Ozen S, Doganci EB, Ozyuvali A, Yalcin AP. Effectiveness of continuous versus pulsed short-wave diathermy in the management of knee osteoarthritis: A randomized pilot study. Caspian $\mathrm{J}$ Intern Med. 2019;10:431-8.

16. Kim H, Suzuki T, Saito K, Kim M, Kojima N, Ishizaki $\mathrm{T}$, et al. Effectiveness of exercise with or without thermal therapy for community-dwelling elderly Japanese women with non-specific knee pain: a randomized controlled trial. Arch Gerontol Geriatr. 2013;57:352-9.

17. Akyol Y, Durmus D, Alayli G, Tander B, Bek Y, Canturk F, et al. Does short-wave diathermy increase the effectiveness of isokinetic exercise on pain, function, knee muscle strength, quality of life, and depression in the patients with knee osteoarthritis? A randomized controlled clinical study. Eur J Phys Rehabil Med. 2010;46:325-36.

18. Laufer Y, Zilberman R, Porat R, Nahir AM. Effect of pulsed short-wave diathermy on pain and function of subjects with osteoarthritis of the knee: a placebo-controlled double-blind clinical trial. Clin Rehabil. 2005;19:255-63.

19. Lavelle ED, Lavelle W, Smith HS. Myofascial trigger points. Anesthesiol Clin. 2007;25:841-51.

20. TRAVELL J, RINZLER SH. The myofascial genesis of pain. Postgrad Med. 1952;11:425-34.

21. Simons D, Travell J, Simons L. Travell \& Simons' Myofascial Pain and Dysfunction: The Trigger Point Manual. 2nd ed. Philadelphia: Williams \& Wilkins; 1999.

22. Dor A, Kalichman L. A myofascial component of pain in knee osteoarthritis. J Bodyw Mov Ther. 2017;21:642-7.
23. McCray RE, Patton NJ. Pain relief at trigger points: a comparison of moist heat and shortwave diathermy. J Orthop Sports Phys Ther. 1984;5:175-8.

24. Lee HR, Shim JH, Oh DW. Effects of High-frequency Diathermy Integrated into Suboccipital Release on Tenderness and Neck Mobility and Disability in People with Chronic Tension-type Headache. Phys Ther Korea 2017;24:37-47

25. Kwon K, Kim CM, Park SJ. The Effect of Diathermy Treatment through Physiotherapist's Hand on the Pain and Disability Index in Chronic Lumbar Pain Patients. Journal of the Korea Convergence Society. 2018;9:289-94.

26. Kim YJ, Park JH, Kim JH, Moon GA, Jeon HS Effect of High-frequency Diathermy on Hamstring Tightness. Phys Ther Korea. 2021;28:65-71

27. Gallagher EJ, Bijur PE, Latimer C, Silver W. Reliability and validity of a visual analog scale for acute abdominal pain in the ED. Am J Emerg Med. 2002;20:287-90

28. McConnell S, Kolopack P, Davis AM. The Western Ontario and McMaster Universities Osteoarthritis Index (WOMAC): a review of its utility and measurement properties. Arthritis Rheum. 2001;45:453-61.

29. Flansbjer UB, Holmbäck AM, Downham D, Patten C, Lexell J. Reliability of gait performance tests in men and women with hemiparesis after stroke. J Rehabil Med. 2005;37:75-82.

30. Salbach NM, Mayo NE, Higgins J, Ahmed S, Finch LE, Richards CL. Responsiveness and predictability of gait speed and other disability measures in acute stroke. Arch Phys Med Rehabil. 2001;82:1204-12.

31. Kuru T, Yalıman A, Dereli EE. Comparison of efficiency of Kinesio ${ }^{\circledR}$ taping and electrical stimulation in patients with patellofemoral pain syndrome. Acta Orthop Traumatol Turc. 2012;46:385-92.

32. Tao XG, Bernacki EJ. A randomized clinical trial of continuous low-level heat therapy for acute muscular low back pain in the workplace. J Occup Environ Med. 2005;47:1298-306.

33. Valdes K, Marik T. A systematic review of conservative interventions for osteoarthritis of the hand. J Hand Ther. 2010;23:334-51.

34. Van Der Heijden RA, Lankhorst NE, Van Linschoten R, Bierma-Zeinstra SM, Van Middelkoop M. Exercise 
for treating patellofemoral pain syndrome: an abridged version of Cochrane systematic review. Eur J Phys Rehabil Med. 2016;52:110-33.

35. Rabini A, Piazzini DB, Tancredi G, Foti C, Milano $\mathrm{G}$, Ronconi $\mathrm{G}$, et al. Deep heating therapy via microwave diathermy relieves pain and improves physical function in patients with knee osteoarthritis: a double-blind randomized clinical trial. Eur J Phys Rehabil Med. 2012;48:549-59.

36. Hassan BS, Doherty SA, Mockett S, Doherty M. Effect of pain reduction on postural sway, proprioception, and quadriceps strength in subjects with knee osteoarthritis. Ann Rheum Dis. 2002;61:422-8.

37. Messier SP, Glasser JL, Ettinger WH Jr, Craven TE, Miller ME. Declines in strength and balance in older adults with chronic knee pain: a 30-month longitudinal, observational study. Arthritis Rheum. 2002;47:141-8.

38. Wellsandt E, Golightly Y. Exercise in the management of knee and hip osteoarthritis. Curr Opin Rheumatol. 2018;30:151-9.

39. Zhang QB, Zhou Y, Zhong HZ, Liu Y. Effect of Stretching Combined With Ultrashort Wave Diathermy on Joint Function and Its Possible Mechanism in a Rabbit Knee Contracture Model. Am J Phys Med Rehabil. 2018;97:357-63.

40. McGorm H, Roberts LA, Coombes JS, Peake JM. Turning Up the Heat: An Evaluation of the Evidence for Heating to Promote Exercise Recovery, Muscle Rehabilitation and Adaptation. Sports Med. 2018; 48:1311-28.

41. Alburquerque-García A, Rodrigues-de-Souza DP, Fernández-de-las-Peñas C, Alburquerque-Sendín F. Association between muscle trigger points, ongoing pain, function, and sleep quality in elderly women with bilateral painful knee osteoarthritis. J Manipulative Physiol Ther. 2015;38:262-8.

42. Hinman RS, Bennell KL, Metcalf BR, Crossley KM. Balance impairments in individuals with symptomatic knee osteoarthritis: a comparison with matched controls using clinical tests. Rheumatology (Oxford). 2002;41:1388-94.

43. Hall MC, Mockett SP, Doherty M. Relative impact of radiographic osteoarthritis and pain on quadriceps strength, proprioception, static postural sway and lower limb function. Ann Rheum Dis.
2006;65:865-70

44. Kim D, Park G, Kuo LT, Park W. The effects of pain on quadriceps strength, joint proprioception and dynamic balance among women aged 65 to 75 years with knee osteoarthritis. BMC Geriatr. 2018; 18:245.

45. Gezginaslan Ö, Öztürk EA, Cengiz M, Mirzaoğlu T, Çakcı FA. Effects of isokinetic muscle strengthening on balance, proprioception, and physical function in bilateral knee osteoarthritis patients with moderate fall risk. Turk $\mathrm{J}$ Phys Med Rehabil. 2018;64:353-61.

46. Witvrouw E, Danneels L, Asselman P, D'Have T, Cambier D. Muscle flexibility as a risk factor for developing muscle injuries in male professional soccer players. A prospective study. Am J Sports Med. 2003;31:41-6.

47. Hurwitz DE, Hulet CH, Andriacchi TP, Rosenberg AG, Galante JO. Gait compensations in patients with osteoarthritis of the hip and their relationship to pain and passive hip motion. J Orthop Res. 1997; 15:629-35.

48. Debi R, Mor A, Segal G, Segal O, Agar G, Debbi E, et al. Correlation between single limb support phase and self-evaluation questionnaires in knee osteoarthritis populations. Disabil Rehabil. 2011;33:1103-9.

49. Mündermann A, Dyrby CO, Hurwitz DE, Sharma L, Andriacchi TP. Potential strategies to reduce medial compartment loading in patients with knee osteoarthritis of varying severity: reduced walking speed. Arthritis Rheum. 2004;50:1172-8.

50. Robon MJ, Perell KL, Fang M, Guererro E. The relationship between ankle plantar flexor muscle moments and knee compressive forces in subjects with and without pain. Clin Biomech(Bristol, Avon) 2000;15:522-7.

51. Liu MQ, Anderson FC, Schwartz MH, Delp SL. Muscle contributions to support and progression over a range of walking speeds. $\mathrm{J}$ Biomech. 2008;41:3243-52.

52. van Dijk GM, Veenhof C, Schellevis F, Hulsmans $\mathrm{H}$, Bakker JP, Arwert H, et al. Comorbidity, limitations in activities and pain in patients with osteoarthritis of the hip or knee. BMC Musculoskelet Disord. 2008;9:95.

53. Lewek MD, Rudolph KS, Snyder-Mackler L. 
Quadriceps femoris muscle weakness and activation failure in patients with symptomatic knee osteoarthritis. J Orthop Res. 2004;22:110-5.

54. Suh MJ, Kim BR, Kim SR, Han EY, Nam KW, Lee SY, et al. Bilateral Quadriceps Muscle Strength and Pain Correlate With Gait Speed and Gait Endurance Early After Unilateral Total Knee Arthroplasty: A Cross-sectional Study. Am J Phys Med Rehabil. 2019;98:897-905. 